\title{
Regenbach und die Babenberger
}

\author{
von BERNHARD BIEDERMANN
}

Rätsel wollen gelöst werden, gerade die, welche als unlösbar gelten. Seitdem der Landeshistoriker Eugen Gradmann das Schlagwort vom „Rätsel von Regenbach"1 geprägt hat, haben sich im Laufe der letzten hundert Jahre Generationen von Pfarrern, Archäologen, Kunstgeschichtlern und Historikern an der Lösung der Frage versucht, wozu das heute unscheinbare Dorf Unterregenbach am Ufer der Jagst einmal gedient haben könnte. Karolingerzeitliche Funde deuten auf einen kleinen Herrensitz, entstanden um 800, der dann Anfang des 11. Jahrhunderts für Größeres gedacht war, als dort eine großzügig bemessene Basilika von 48 m Länge und 17 m Breite entstand. Zum Vergleich: Die Stadtkirche St. Michael der stauferzeitlichen Salzstadt Schwäbisch Hall hatte im ausgehenden 12. Jahrhundert die Maße $53 \mathrm{~m} \mathrm{x} 18 \mathrm{~m} .^{2}$ Es hat sich zudem eine Urkunde vom 9. August 1033 erhalten, in welcher dokumentiert ist, dass damals Kaiserin Gisela und Kaiser Konrad II. Regenbach an das Bistum Würzburg geschenkt haben. ${ }^{3}$ Im Verbrüderungsbuch der Abtei Reichenau, welches ab dem 9. Jahrhundert jene adeligen Gönner auflistet, die sich durch finanzielle Zuwendungen das immerwährende Gebetsgedenken der Reichenauer Mönche sicherten, stoßen wir nach meiner Ansicht auf jene Sippe, deren Familiengeschichte uns nach Regenbach an der Jagst führt:

LUITPOLD MARCHIUS - RIHUUAR - JUDITA - HEINRICH - ERNUST POPPO - LUITPOLD - CUNIGUNT - ADALBR: - LUITPOLD PRESBYTER - HEINRICH. ${ }^{4}$

Bei dem Markgrafen Luitpold und Richwara (oder Richardis) handelt es sich um die Eltern. Aus der Schar der hier aufgelisteten Nachkommen sind für unsere Geschichte von Bedeutung die Söhne Ernust (Ernst), Poppo und Adalbr(aht) (Adalbert). Der Eintrag auf der Reichenau dürfte um 980 erfolgt sein.

1 Eugen Gradmann: Das Rätsel von Regenbach. In: WVjH NF 25 (1916), S. 1-46.

2 Hans Werner Hönes: Die Baugeschichte. In: Historischer Verein für Württembergisch Franken u. a. (Hg.): Sankt Michael in Schwäbisch Hall. Künzelsau 2006, S. 76-115, hier S. 79.

3 Peter Hilsch: Regenbach und die Schenkung der Kaiserin Gisela. In: ZWLG (1983), S. 52-82.

4 Heide Dienst: Werden und Entwicklung der Babenbergischen Mark. In: Anna M. Drabek, Richard G. Plaschka (Hg.): Österreich im Hochmittelalter - 907 bis 1246 (Veröffentlichungen für die Geschichte Österreichs 17). Wien 1991, S. 63-102, hier S. 76. 
Luitpold von Schweinfurt ist erstmals am 21. Juli 976 als Markgraf der von Baiern abgetrennten Ostmark genannt, ${ }^{5}$ einer Region etwa zwischen Linz und Wien, für die seit 996 auch die Bezeichnung Ostarrichi belegt ist, ${ }^{6}$ die Keimzelle des späteren Österreich. Das Leben Luitpolds wurde ausgelöscht, als ihn 994 beim Besuch des Kilianifestes in Würzburg ein Pfeil traf, der eigentlich seinen Vetter Hezilo (Heinrich) von Schweinfurt töten sollte. Die Ehefrau Richwara entstammte der Sippe der Ernestinger, ${ }^{7}$ die seit dem 9. Jahrhundert zunächst den baierischen Nordgau, dann den westlich anschließenden fränkischen Sualafeld-Gau mit Sitz in Weißenburg (Mittelfranken) verwaltet hat. Da dem Nordgau-Grafen Ernst I. († 865) von Kaiser Ludwig dem Frommen das Kammergut Lauffen am Neckar anvertraut worden war, ${ }^{8}$ ist es naheliegend, dass auch die beiden zwischen dem Neckar im Westen und dem Sualafeld im Osten gelegenen Grafschaften des Kocher- und Maulachgaus in die Zuständigkeit der Ernestinger fielen. Jedenfalls sind in diesen Zeiten keine Namen von Kochergau- und Maulachgau-Grafen überliefert. Natürlich würden sie sich in einem solchen Fall von Untergrafen vor Ort vertreten haben lassen. Sitz eines solchen Vertreters könnte im 9. und 10. Jahrhundert Regenbach gewesen sein, das unmittelbar am Übergang vom Maulachgau in den Kochergau gelegen war. Die vom Familiensitz Weißenburg weit entfernte Lage von Regenbach spricht dafür, dass dieser Ort später zum Heiratsgut der Richwara gehörte. Man hat abseits gelegene Punkte gerne den Töchtern in die Ehe mitgegeben, da sie für die Ursprungsfamilie zwar verloren, der Verlust aber leichter zu verschmerzen war, als wenn man vom zentralen Familiengut einen Teil hätte abgeben müssen.

Nach dem plötzlichen Tod Markgraf Luitpolds trat der älteste Sohn Heinrich dessen Nachfolge in der Ostmark an mit Sitz in Melk an der Donau. ${ }^{9}$ Die fränkischen Güter gingen an den zweitgeborenen Sohn Ernst, der seinen Sitz zunächst in Aura an der Fränkischen Saale nördlich von Schweinfurt hatte. ${ }^{10}$ Der dritte Sohn Poppo schlug eine geistliche Laufbahn ein, die ihn von der Domschule Regensburg über das Amt des Propstes am Dom zu Bamberg schließlich 1016 auf den Sitz des Erzbistums Trier führte. ${ }^{11}$ Der nachgeborene Sohn Adalbert übernahm zunächst Aufgaben im fränkischen Radenzgau, dann in baierischen Grafschaften an der Donau zwischen Regensburg und Passau. ${ }^{12}$ Als sein ältester

5 Friedrich Stein: Markgraf Luitpold von Österreich und seine Söhne. In: Archiv des Historischen Vereins für Unterfranken 42 (1900), S. 53-56, hier S. 54.

6 Karl Lechner: Die Babenberger. Markgrafen und Herzöge von Österreich. Wien u. a. 1976, S. 58.

7 Franz Tyroller: Die Ahnen der Wittelsbacher zum anderen Male. In: Jahrbuch für Fränkische Landesforschung 15 (1955), S. 129-156, hier S. $148 \mathrm{ff}$.

8 Hansmartin Schwarzmaier: Die Reginswindis-Tradition von Lauffen. In: ZGO 131 (1983). Festschrift für Gerd Tellenbach (1983), S. 163-198.

9 Lechner (wie Anm. 6), S. 63.

10 Friedrich Stein: Geschichte Frankens. Band I: Das Mittelalter. Schweinfurt 1885, S. 142.

11 Vgl. Wolfgang Schmid: Poppo von Babenberg († 1047), Erzbischof von Trier - Förderer des heiligen Simeon - Schutzpatron der Babenberger. Trier 1998.

12 Lechner (wie Anm. 6), S. 62-65. 
Bruder Markgraf Heinrich der Ostmark bereits 1018 ohne Nachkommen verstarb, trat Adalbert dort die Nachfolge an und wurde zum eigentlichen Stammvater der österreichischen Babenberger, die bis 1246 die Entwicklung Österreichs maßgeblich bestimmten. Die Bezeichnung „Babenberger“ ist von Bischof Otto von Freising geprägt, der in seiner „Chronica“ sein väterliches Geschlecht bis zu Markgraf Adalbert (1018-1055) zurückführt ${ }^{13}$ und einen ehemaligen Sitz der Familie - die Babenburg (Bamberg) - als Namensgrundlage heranzieht.

Für kurze Zeit sah es so aus, als stünde dem Zweig der fränkischen Babenberger eine glänzende Zukunft bevor. Um das Jahr 1008 - der genaue Zeitpunkt ist unbekannt - heiratete Ernst die verwitwete Gisela von Schwaben, Tochter Herzog Hermanns II. von Schwaben. Drei Kinder wurden geboren: Ernst, Gisela, Hermann. Überraschend starb 1012 Giselas Bruder, Herzog Hermann III. von Schwaben. So ging das Herzogsamt an ihren Ehemann Ernst. Seinen Herrenhof Aura in der Vorrhön schenkte Herzog Ernst I. (1012-1015) an das Bistum Bamberg, welches dort später ein Benediktinerkloster errichtete. Die Verpflichtungen in Schwaben verlagerten offenbar die Interessen Richtung Süden. Der Besitz Regenbach, der aus dem Gut der Mutter Richwara in das Eigentum des in Franken und Schwaben tätigen Sohnes übergegangen sein dürfte, könnte nun eine größere Aufmerksamkeit erfahren haben. Für zukünftige Pläne lag der Ort am Übergang Frankens nach Schwaben nun ziemlich günstig. Der plötzliche Tod Herzog Ernsts I. im Frühsommer $1015^{14}$ aber machte eventuelle Pläne für Regenbach zunichte. Auf einer Jagd wurde er versehentlich von einem Pfeil getroffen, der einem im Dickicht verfolgten Hirsch gegolten hatte. Sterbend bat Ernst noch darum, in Würzburg an der Seite seines Vaters beigesetzt zu werden.

Es hat in Regenbach bis zu diesem Zeitpunkt vermutlich noch keinerlei Aktivitäten zum Ausbau des Platzes an der Jagst gegeben. Denn es ist zu beobachten, dass Schenkungen aus dem Umkreis der Babenberger und Ernestinger damals vorzugsweise an das Kloster Sankt Emmeram in Regensburg gingen. Graf Ernst V., Bruder Richwaras, der ohne männliche Nachkommen verstorbene letzte Ernestinger aus Weißenburg, lag dort begraben. Seine Witwe Pilifridis beschenkte vorzugsweise Sankt Emmeram. ${ }^{15}$ Auch Schenkungen des Schweinfurter Markgrafen Berthold, seiner Frau Eilika und seines Sohnes Hezilo sind zuvor dorthin gegangen. ${ }^{16}$ Hätte es um die Jahrtausendwende schon Pläne für Regenbach gegeben, wären Schenkungen aus dem Umkreis der Babenberger und Ernestinger wohl eher für Regenbach reserviert worden. Auch Bischof Thietmar von Merse-

13 Ebd., S. $40 \mathrm{ff} .$.

14 Gerd Wunder: Gisela von Schwaben. In: Robert Uhland (Hg.): Lebensbilder aus Schwaben und Franken XIV. Stuttgart 1980, S. 1-16.

15 Christine Rädlinger-Prömper: Sankt Emmeram in Regensburg - Struktur- und Funtionswandel eines bayerischen Klosters im frühen Mittelalter (Thurn und Taxis-Studien 16). Kallmünz 1987, S. 216.

16 Ebd., S. 222-225 (Trad. 210). 
burg, ${ }^{17}$ der als Neffe Eilikas von Schweinfurt viele Berichte über die Babenberger hinterlassen hat, erwähnt in seinen Aufzeichnungen, die 1018 enden, Regenbach mit keinem Wort.

Kurz danach aber muss es eine Entscheidung für den Ausbau Regenbachs gegeben haben. Zwei hochrangige Mitglieder der Familie - Markgraf Luitpold $(†$ 994) und Herzog Ernst I. († 1015) - waren unvorbereitet, ohne Sterbesakramente, ums Leben gekommen. Nur der Papst hätte ,aus apostolischer Autorität“ den Toten im Nachhinein die Absolution erteilen können. ${ }^{18}$ Der Familie oblag es nun, für eine angemessene „Memoria“ zu sorgen, am besten durch die Errichtung einer Memorialkirche, in der ein immerwährendes Gebetsgedenken dem Seelenheil der Verstorbenen zugutekäme. Im Falle der fränkischen Babenberger ruhte die Verantwortung für die Errichtung einer Memorialstiftung nun in den Händen des ältesten Sohnes Ernst und - solange dieser noch unmündig war seines Onkels und Vormunds ${ }^{19}$ Erzbischof Poppo von Trier.

Die Witwe Gisela hatte 1016 ihre dritte Ehe geschlossen, diesmal mit Konrad von Worms. Eine Memoria für ihren zweiten Ehemann und ihren einstigen Schwiegervater, den sie nie kennengelernt hatte, war von ihr nicht zu erwarten. Regenbach an der Jagst hat sie womöglich nie gesehen.

Erzbischof Poppo von Trier (1016-1047) halte ich für die entscheidende Persönlichkeit, der Regenbach zu verdanken ist. Nach der Gründung des Bistums Bamberg 1007 hatte er als Vorsteher des dortigen Domkapitels Erfahrungen in der Errichtung der Gründungsbauten sammeln, Kontakte zu kundigen Bauleuten aufbauen und damit ab etwa 1020 die Errichtung der großen Basilika in Regenbach vorantreiben können. Seit der Mitte der 1030er Jahre flossen diese Erfahrungen in seiner Bischofsstadt Trier in enorme städtebauliche Veränderungen ein, wo er innerhalb weniger Jahre eine Krypta unter dem Hochaltar des Domes, eine Erweiterung der Bischofskirche nach Westen sowie den Umbau des römerzeitlichen Stadttores Porta Nigra zu einer Stiftskirche zum Gedenken an seinen 1041 heiliggesprochenen Freund Simeon ${ }^{20}$ vornehmen ließ. In der Simeons-Stiftskirche fand er nach seinem plötzlichen Tod, der ihn am 16. Juni 1047 auf der Dombaustelle zu Trier ereilte, seine letzte Ruhestätte.

Nach dem tödlichen Jagdunfall Herzog Ernsts I. 1015 war das Herzogtum Schwaben an dessen damals etwa 7-jährigen Sohn Ernst II. übertragen worden unter der Vormundschaft seines Onkels Poppo von Trier. Spätestens im Jahr 1024 war Ernst II. von Schwaben dann mündig, denn seit diesem Jahr sind eigenverantwortliche Handlungen des nun etwa 16-jährigen urkundlich belegt. Dass er Besitz in der Region an Jagst und Kocher gehabt haben muss, erschließt

17 Werner Goez: Bischof Thietmar von Merseburg, Geschichtsschreiber. In: Werner Goez: Lebensbilder aus dem Mittelalter. Die Zeit der Ottonen, Salier und Staufer. Darmstadt 2010, S. $106 \mathrm{ff}$.

18 Lechner (wie Anm. 6), S. 138.

19 Wunder (wie Anm. 14), S. 4.

20 Dienst (wie Anm. 4), S. 82. 
sich aus der Verleihung eines Wildbanns für die Abtei Ellwangen. ${ }^{21}$ Kaiser Heinrich II. ließ Herzog Ernst II. als einzigen der vom Vorgang betroffenen Anrainer in der Urkunde vom 5. Februar 1024 namentlich erwähnen, indem betont wurde, der Wildbann sei mit ausdrücklicher Zustimmung von Ernst an Ellwangen vergeben worden. ${ }^{22}$ Der blutjunge Babenberger war auch beteiligt an der Königswahl zu Kamba am Rhein, als dort am 7. September 1024 sein Stiefvater zum König der Deutschen gewählt ${ }^{23}$ und tags darauf in Mainz als König Konrad II. gekrönt wurde.

Die Aufgabe als Nachfolger des Vaters im Amt eines Herzogs von Schwaben könnte auch für die Entwicklung von Regenbach von Bedeutung gewesen sein. Ursprünglich als Memorialkirche für Vater und Großvater geplant, deuten die Ausmaße der großen Basilika darauf hin, dass sich hier auch eine repräsentative Grablege der fränkischen Babenberger entwickeln sollte. Die österreichischen Babenberger haben gerade in dieser Zeit Melk an der Donau als Erbbegräbnis vom Burgensitz zum Kollegiatstift umgewandelt. ${ }^{24}$ Es ist naheliegend, das Bauprojekt von Regenbach auch unter diesem Aspekt zu betrachten. Großartige Pläne aber scheitern für gewöhnlich an der Wirklichkeit.

Die folgenden Jahre - es sind die letzten im kurzen Leben des Herzogs Ernst II. - sind geprägt von anhaltenden Spannungen zwischen ihm und König Konrad II. Aufstand, Versöhnung, erneute Revolte, erneute Versöhnung. Jedesmal scheint es Gisela gewesen zu sein, die zwischen Herzog und König, zwischen Stiefsohn und Stiefvater vermitteln konnte. Doch dann, beim dritten Aufstand, konnte oder wollte selbst sie nichts mehr retten.

1028 oder 1029 war Ernst aus vorübergehender Inhaftierung entlassen worden. ${ }^{25}$ Das Amt eines Herzogs von Schwaben hatte man ihm nach seinem zweiten Aufstand entzogen, ihm bei seiner Freilassung aber das Herzogtum Baiern in Aussicht gestellt. Für diese Verleihung sollte er den vom Vater und der Großmutter Richwara ererbten Familiensitz Weißenburg im Sualafeld-Gau an den inzwischen zum Kaiser gesalbten Konrad II. abtreten. Nach etwa einjähriger Bewährungsfrist sah es auf einem Hoftag zu Ingelheim im April 1030 sogar danach aus, als könnte Ernst wieder in sein früheres Herzogtum Schwaben eingesetzt werden. Die Forderung, dann aber auch aktiv gegen seinen Freund und ehemaligen Vasallen Werner von Kyburg vorgehen zu sollen, überforderte die Langmut des jungen Mannes. Erneut richtete sich seine Wut gegen Kaiser Konrad II. Der rückfällig Gewordene wurde ,,in den Bann getan und mit dem Verlust aller seiner

21 Hermann Rettenmeier: Die Grenzen des Ellwanger Bannforsts in der Zeit des altdeutschen Kaisertums. In: Ellwanger Jahrbuch 15 (1950-1953), S. 72-125.

22 Otto Hutter: Das Gebiet der Reichsabtei Ellwangen (Darstellungen aus der württembergischen Geschichte 12). Stuttgart 1914, S. 15.

23 Christoph Friedrich Stälin: Wirtembergische Geschichte Band 1. Erster Theil: Schwaben und Südfranken von der Urzeit bis 1080. Stuttgart, Tübingen 1841, S. 476.

24 Dienst (wie Anm. 4), S. 86.

25 Stälin (wie Anm. 23), S. 481. 
Güter bestraft". Das für ihn erneut vorgesehene Amt eines Herzogs von Schwaben ging stattdessen an seinen jüngeren Bruder Hermann. In den nun folgenden blutigen Auseinandersetzungen fand Ernst II. zusammen mit Werner von Kyburg und anderen Mitstreitern am 17. August 1030 den Tod.

In Regenbach wird es nach diesen Vorgängen zu einem Stillstand gekommen sein. Als eingezogenes Gut war es nun an Kaiser Konrad II. zu entscheiden, was damit geschehen sollte. Üblich war es, nach einer Schamfrist solchen Besitz wieder an die Sippe des einstigen Delinquenten zu restituieren. Der Herrscher war auf die Unterstützung der weitverzweigten Adelssippen angewiesen. Nun traf es sich, dass Kaiser Konrad II. mit der Mutter des Bestraften verheiratet war. Was lag näher, als das ehemalige Gut Ernsts II. nun in die Hände seiner Mutter zu übertragen? Ich denke, dass Kaiserin Gisela auf diesem Wege an Regenbach gekommen ist. Aber nur für kurze Zeit, denn schon bald gingen proprietas und hereditas, ${ }^{26}$ gingen Nutzungsrecht und Freies Eigen an der Jagst in den Besitz des Bistums Würzburg oder genauer: in den Besitz des Bistumsheiligen Kilian über.

Schon Kaiser Heinrich II. († 1024) war daran gelegen, die Position des Bistums südlich der Tauber zu stärken. Er hatte Würzburg seinen östlichen Bereich genommen, um dort 1007 das Bistum Bamberg gründen zu können. „Zur Entschädigung [...] erhielt der Bischof von Würzburg [...] die Verleihung der geistlichen Amtsgewalt in den Gauen Wingardeiba sowie im Jagst- und ganzen Kocher- und Neckargau, deren jene bisher zum Bistum Worms, diese zu dem von Speier zuständig war.“'27 Diese Politik der Förderung des Bistums Würzburg im Süden setzte der Amtsnachfolger Konrad II. fort. Ein Baustein dazu war die Verleihung eines Wildbanns rund um Kloster Murrhardt im Jahr 1027, ${ }^{28}$ ein weiterer Baustein die Schenkung eines Teiles von Regenbach im Sommer 1033. Natürlich hätte der Kaiser das eingezogene Gut Ernsts II. auch direkt an Würzburg geben können, aber das schickte sich nicht. Das ehemalige Gut eines im Bann Verstorbenen direkt an den heiligen Kilian? Noch dazu im Jahr des Milleniums der Passion des Herrn Jesus Christus? Da wählte man doch lieber den Umweg einer kreativen Grundstückswäsche.

Vorausschauend war, dass man für dieses Rechtsgeschäft die Zustimmung all jener einholte, die ursprünglich ein Anrecht auf das Gut gehabt hatten als teilberechtigte Erben des Herzogs Ernst II. Niemand sollte jemals die Schenkung an den Heiligen Kilian anfechten. Eine Übersicht zeigt die Zeugen aus der näheren und entfernteren Verwandtschaft Ernsts II., die eigens nach Limburg (Pfalz) zur Beurkundung anreisen mussten:

26 Rädlinger-Prömper (wie Anm. 15), S. 119 (Anm. 658).

27 Peter Paul Albert: Das Bistum Würzburg und die Gaugrafschaft Wingardeiba. In: Würzburger Diözesangeschichtsblätter 14/15 (1952/53), S. 123-139, hier S. 137 ff.

28 Gerhard Fritz: Kloster Murrhardt im Früh- und Hochmittelalter (FWFr 18). Sigmaringen 1982, S. $71 \mathrm{ff}$. 
Ernestinger

Grafen im Sualafeldgau

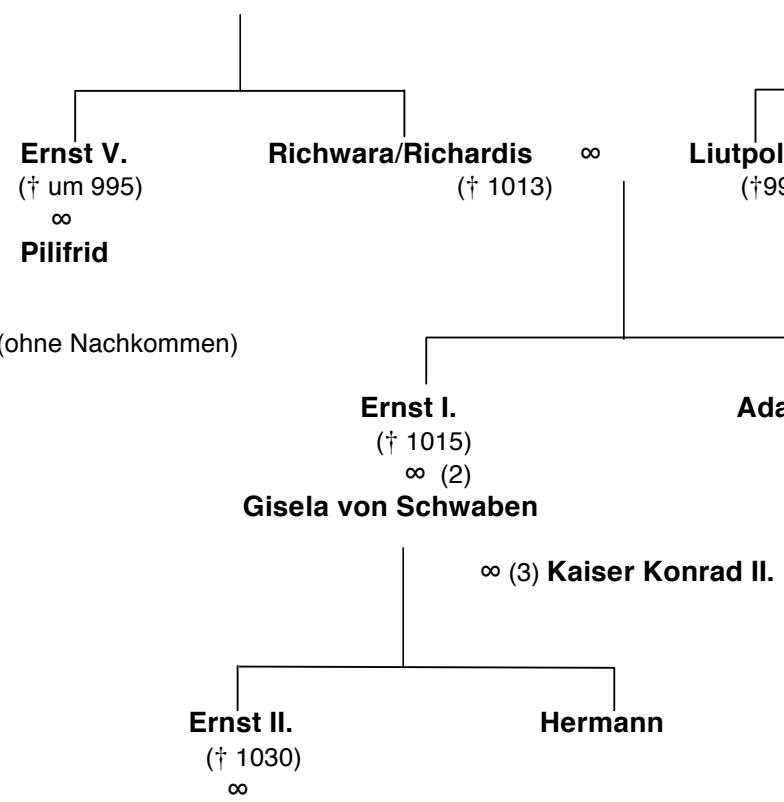

Babenberger

Markgrafen von Schweinfurt

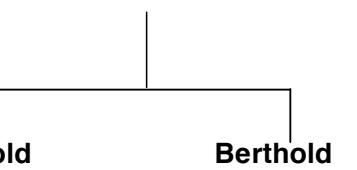

†994)

(† um 980)

$\infty$

Eilika

(† 1015)

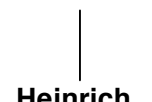

$(\dagger 1017)$

$\infty$

\section{Gerberga}

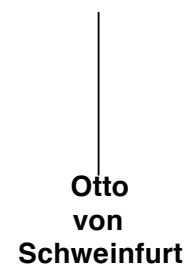

Irmelgard

In der Urkunde vom 9. August $1033^{29}$ sind sie aufgelistet:

\section{Chuono}

Es ist Konrad/Kuno aus der Sippe der lothringischen Pfalzgrafen. ${ }^{30}$ Er zeugt als ältestes männliches Mitglied seines Familienzweigs stellvertretend für seine Schwester Irmelgarda, die Witwe Herzog Ernsts II.

\section{Otto und sein Sohn Udo}

Es ist Otto von Hammerstein, der Bruder von Gerberga von Schweinfurt. Für sie bezeugt er den Verzicht. Gewissermaßen als „Nachrücker“, als baldiger Nachfolger des betagten Vaters bezeugt dies auch dessen Sohn Udo.

3. Ezzo, der Pfalzgraf, und sein Sohn Otto

Es ist der Großvater von Herzog Ernsts Witwe Irmelgarda. Nachdem Chuono bereits für den Zweig Ludolf - Heinrich - Konrad Verzicht geleistet hat, erklären der hochbetagte Ezzo ${ }^{31}$ und sein Sohn Otto den Verzicht für die anderen Zweige der Sippe.

\section{Hilsch (wie Anm. 3), S. 52 ff.}

30 Ruth Gerstner: Die Geschichte der Lothringischen und Rheinischen Pfalzgrafschaft von ihren Anfängen bis zur Ausbildung des Kurterritoriums Pfalz (Rheinisches Archiv 40). Bonn 1941.

31 Walter Bader: Die Benediktinerabtei Brauweiler bei Köln. Berlin 1937. 
4. Otto von Schweinfurt

Sein Onkel mütterlicherseits Otto von Hammerstein hatte bereits den Verzicht für eventuelle Ansprüche über Ottos Mutter Gerberga erklärt. Nun zeugt deren Sohn Otto von Schweinfurt für sich selbst und eventuelle Nachkommen.

\section{Markgraf Adalbert}

Der Onkel Herzog Ernsts II. bezeugt den Verzicht für die Linie der österreichischen Babenberger.

6. Der danach in der Urkunde erwähnte Graf Eberhard mit den folgenden Einzelnamen dürfte der Vogt des Würzburger Kirchenguts ${ }^{32}$ sein, der auch bei späteren Rechtsgeschäften des Bistums Würzburg zeugt. Seine Anwesenheit und die seiner Leute ist notwendig, da sie ja wissen müssen, worum sie sich zukünftig zu kümmern haben.

7. Der dann genannte Herzog Hermann ist der bisherige Vogt, der das Gut seiner Mutter geschützt hat. Die ihm nachstehend genannten vier Einzelnamen dürften die Namen jener Leute sein, die bisher vor Ort für das Gut tätig waren. Sie zeugen, damit sie genau wissen, was sie zukünftig nichts mehr angeht.

Die Urkunde betont, dass man nur einen Teil der zum einstigen geistlichen Besitz von Regenbach an den Würzburger Bistumsheiligen in dieser Form übergibt. Vermutlich ist damit der Kern, das heutige Dorf Unterregenbach mit seinen damaligen Sakralbauten gemeint. Der andere Teil - Güter in der Region - dürfte ohne große Umstände entweder als Lehen oder gar als Freies Eigen direkt von Kaiser Konrad II. an treue Gefolgsleute gegangen sein. Die Herren von Mulfingen, von ihnen abzweigend die späteren Herren von Krautheim und Herren von Langenberg, sind vermutlich als erste in den Genuss der Umverteilung gekommen. Ihr Besitz in dieser Ecke des Landes wird aber erst ab dem Ende des 11. Jahrhunderts nachvollziehbar.

Ist die Urkunde 1033 das aussagekräftigste schriftliche Zeugnis von der frühen Geschichte Regenbachs, so ist die weitgehend erhaltene Krypta der einstigen großen Basilika das anschaulichste Baudenkmal, welches uns noch heute von diesen fernen Zeiten kündet. Kunsthistoriker haben darauf hingewiesen, dass die hiesige Krypta mit ihren Pyramidenstumpfkapitellen auf Vierkantpfeilern, die Tonnengewölbe mit Stichkappen tragen, große Ähnlichkeit hat mit den in der ersten Hälfte des 11. Jahrhunderts entstandenen Krypten von Roßtal (Mittelfranken) und Bleurville (Lothringen). Die verblüffenden Übereinstimmungen lassen den Schluss zu, dass bei diesen Bauprojekten dieselben Ideengeber im Hintergrund tätig gewesen sein könnten.

In der Person des Erzbischofs Poppo von Trier lassen sich Verbindungslinien zu allen drei Projekten aufzeigen. Seine Rolle im Zusammenhang mit dem Bau der 
Krypta von Regenbach ist schon benannt. In Roßtal dürfte er zusammen mit Herzog Ernsts II. Witwe die treibende Kraft gewesen sein.

Historische Vereine sind Kinder der Romantik. Nachdem Reichsfreiherr vom und zum Stein 1819 die „Gesellschaft für ältere deutsche Geschichtskunde“ gegründet hatte, entstanden in den folgenden Jahren auf regionaler Ebene Historische Vereine als Zusammenschlüsse Gleichgesinnter, die mehr oder weniger systematisch damit begannen, die Erinnerungen an geschichtliche Ereignisse ihrer Region wachzuhalten oder dort, wo sie verschüttet waren, diese zu wecken. Die Monarchen förderten dieses Interesse an der Historie, weil man - wie König Ludwig I. von Bayern verkünden ließ - vom ,hohen Werthe“ des „Studiums der Vergangenheit für die Gegenwart“ überzeugt war. So die offizielle Version, die noch heute gerne bei Tagungen bayerischer Heimatpfleger geflötet wird. Inoffiziell freilich begründeten die Berater des Königs dessen Denkmalpflege-Erlass von 1827 damit, „,dass die Historie ein spezifisches Gegengift wider revolutionäre Neuerung und wider ungeduldiges Experimentieren sey, - wer seinen Sinn ernst und würdig auf die Vergangenheit richte, sey nicht zu fürchten in der Gegenwart". ${ }^{33}$ Sollen die Leute doch ihre Nase in den Plunder alter Ritterburgen stecken, dann fehlt ihnen wenigstens die Zeit, sich Gedanken zu machen über Verfassungen und Bürgerrechte und womöglich sogar demokratische Beteiligung an politischen Veränderungen.

Mit dem Segen des Monarchen entstanden in den Jahren 1830/31 in den bayerischen Kreisen Historische Vereine, welche die Ergebnisse ihrer Forschungen in Jahresberichten vorlegten. Schon 1838 findet sich da die Notiz eines Bibliothekars Huscher aus Ansbach: „Dass in der Kirche zu Roß-Stall die Epitaphien eines gewissen Herzogs Ernst und seiner Gemahlin Irmelgard einst wirklich vorhanden waren, lässt sich [...] nicht leicht bezweifeln." Und er zitiert die Beschriftung des Epitaphs mit A SACTA ERBELGARDE RHENI PALATINISSA Sowie ERNESTINE VERO, BAVARORUM DUCIS. ${ }^{34}$

Auf diese Notiz des Ansbacher Bibliothekars bezieht sich Stälin, wenn er später vermerkt: „Ernsts Leiche wurde nach Constanz gebracht und nach Lösung des Bannes in der dortigen St. Marienkirche, später in dem Familienbegräbnis zu Roß-Stall [...] beigesetzt." 35

Die Witwe Herzog Ernsts, auf welche die Roßtals-Kirche mit der romanischen Krypta zurückgeht, ist Irmelgard aus der Familie der lothringischen Pfalzgrafen. Dass sie auf dem Epitaph fälschlicherweise als „Rheinische Pfalzgräfin“ bezeichnet wurde, zeigt, dass dieses erst Jahrzehnte nach ihrem Tod entstanden sein kann. Die lothringische Pfalzgrafschaft war im Raum Aachen-Köln ange-

33 Gerd-Helge Vogel (Hg.): Julius Schnorr von Carolsfeld und die Kunst der Romantik. Publikation der Beiträge zur VII. Greifswalder Romantikkonferenz in Schneeberg 1994. Greifswald 1996.

34 Neunter Jahresbericht des Historischen Vereins in Mittelfranken (1838), [ohne Verfasser]: § 3: Eingesendete Abhandlungen, Handschriften und Urkunden, S. 12-33, hier S. 24 unter Ziffer 13: „Von Herrn Bibliothekar Huscher in Ansbach“, dessen Bericht zu Roßtal: S. 24-31.

35 Stälin (wie Anm. 23), S. 482. 
siedelt, wandelte sich erst nach und nach durch den Wechsel vom Niederrhein über die Moselgegend an den Mittelrhein zur Rheinischen Pfalzgrafschaft. Der erste Pfalzgraf, der sich Comes Palatinus Rheni nannte, war Heinrich von Laach $(† 1095)$, Stifter der Abtei Laach und Förderer der Komburg. ${ }^{36}$

Irmelgard dürfte identisch sein mit jener Frau Yrmengarda, die zwischen 1024 und 1040 in Urkunden des Bistums Bamberg genannt wird. ${ }^{37}$ Roßtal, Fürth, Langenzenn, Herzogenaurach, Sebalder Reichswald sind Orte, in denen sie über ansehnlichen Besitz verfügt. Das ist Königsgut, kein Gut, welches man auf die Ernestinger zurückführen könnte. ${ }^{38}$ Irmelgards Großvater Ezzo, der in der Urkunde zu Regenbach 1033 als Zeuge auftritt, war mit Mathilde, der Schwester Kaiser Ottos III., verheiratet. Über diese Großmutter dürfte liudolfingisches Erbgut des Königshauses an Irmelgard gelangt sein, darunter Roßtal. Ihre angeheiratete Verwandtschaft mit den Ernestingern blitzt noch einmal auf an unerwarteter Stelle. Im Nekrolog des Klosters Einsiedeln in der Innerschweiz gibt es einen Eintrag, der offenbar mehreren miteinander familiär verbundenen Menschen gilt. ${ }^{39}$

COM(ES) UOLRICUS DE BAVARIA, RICHKARTA UXOR EIUS. HESSO, GISELA. DOMINA IRMENGARDIS, MATER HERMANNI ABBATIS.

Graf Ulrich von Ebersberg $(\dagger 1029)$ und seine Ehefrau Richardis $(\dagger 1013)$ sind auch mit Schenkungen an das Kloster Sankt Emmeram in Regensburg belegt. ${ }^{40}$ Ich bin überzeugt, dass es sich bei Richardis um Richwara handelt, die nach dem Tod Markgraf Luitpolds von der Ostmark eine zweite Ehe mit dem Ebersberger eingegangen ist, aus dessen Sippe ihre Schwägerin Pilifrid stammte. Gisela und Hesso sind Gisela von Backnang, ${ }^{41}$ Enkelin der Richwara/Richardis, und ihr Ehemann. Irmengardis wäre dann als Witwe Herzog Ernsts die Schwägerin zu Gisela und Hesso. Da sie als Mutter des Abtes Hermann von Einsiedeln bezeichnet wird, wäre dieser Abt möglicherweise ein bisher nicht identifizierter Sohn Herzog Ernsts II. von Schwaben. Er hätte diesen Eintrag im Nekrolog seines Klosters Einsiedeln in Erinnerung an seine Mutter, seine Tante Gisela und seine Urgroßmutter Richwara veranlassen können. Abt Hermann († 1065) könnte seinen Namen von seinem Onkel, vielleicht auch Taufpaten, Herzog Hermann IV. von Schwaben (1030-38) erhalten haben, der an maßgeblicher Stelle an der Schenkungsurkunde von Regenbach 1033 beteiligt war. Dessen Tochter Richwa-

36 Gerstner (wie Anm. 30), S. 45.

37 Robert Leyh: Evangelische Stadtkirche Langenzenn und ehemaliges Augustiner Chorherrenstift. Langenzenn 1993, S. 6 f (Anm. 10).

38 Tyroller (wie Anm. 7), S. 152 (Anm. 115).

39 Fritz (wie Anm. 28), S. 130 (Anm. 28).

40 Rädlinger-Prömper (wie Anm. 15), S. 215.

41 Fritz (wie Anm. 28), S. 132. 
ra wurde später durch ihre Ehe mit Bertold I. die Stammmutter der Zähringer und der Markgrafen von Baden.

Irmelgard hat die Kirche von Roßtal sicher mit Unterstützung Erzbischofs Poppo von Trier errichten lassen. Dass dieser nach dem Scheitern von Regenbach an einer würdigen Grabstätte für Ernst interessiert war, ist nachvollziehbar, war er doch lange Jahre Vormund dieses Neffen gewesen. Als Bauzeit würde ich die Jahre zwischen 1030, dem Todesjahr Ernsts, und 1037 ansetzen. Danach ist Erzbischof Poppo mit der städtebaulichen Umgestaltung seiner Bischofsstadt Trier so ausgelastet, dass er für das entfernte Roßtal kaum mehr zur Verfügung gestanden hätte.

Die Inschrift ERNESTINE VERO. BAVARORUM DUCIS des verschwundenen Roßtaler Epitaphs weist auf das letzte Amt, das Herzog Ernst noch vor seinem Tod ausgeübt hat. Freilich wird dies gerne ignoriert und selbst Bosl ${ }^{42}$ behauptet: „Graf Ernst, Schwiegervater König Karlmanns [...] ist zu Roßtal bei Nürnberg begraben". Das ist der Stammvater der Ernestinger aus dem 9. Jahrhundert, Vater auch der seligen Reginswindis von Lauffen/Neckar, also ein Urahn Herzog Ernsts II. Irmelgards Bruder Konrad von Zütphen (†1055) ist 1049 Herzog von Baiern geworden, ${ }^{43}$ ein Amt, das der Graf vom fernen Niederrhein vielleicht deshalb erhielt, weil es schon einmal Jahre zuvor seinem Schwager Ernst zugestanden worden war. Konrad hat eine Tochter des Babenbergers Otto geheiratet, so dass die beiden Zeugen Chuono und Otto von Schweinfurt aus der Urkunde für Regenbach 1033 nun auch direkt familiär verbunden waren.

Die architektonischen Gemeinsamkeiten der Krypten von Regenbach und Roßtal sind auch bezüglich Bleurville (Lothringen) über Erzbischof Poppo von Trier zu erklären. Bleurville lag im Bistum Toul. Das diesem Bistum übergeordnete Erzbistum war Trier. Bischof Bruno von Toul (1026-1051) war von Erzbischof Poppo in sein Amt eingeführt worden. ${ }^{44}$ Ab 1033 waren Erzbischof Poppo und Bischof Bruno als Gesandte Kaiser Konrads II. zusammen tätig. Daraus entwickelte sich eine langjährige Zusammenarbeit. Es ist naheliegend, dass man da auch über Bauprojekte gesprochen, Fachleute weiterempfohlen hat. Noch nach seiner Wahl zum Papst (Leo IX.) hat Bruno sein Bischofsamt in Toul zunächst beibehalten, hat im Sommer 1049 in Sankt Simeon zu Trier das Grab seines 1047 verstorbenen Mitstreiters Poppo besucht ${ }^{45}$ und im folgenden Jahr persönlich Bleurville geweiht. Eine der Schwestern Brunos von Toul war mit Otto verheiratet, der zusammen mit seinem Vater Ezzo in der Urkunde zu Regenbach 1033 als Zeuge genannt ist. Als Otto 1047 gestorben ist, hat Bischof Bruno persönlich die Bestattungsfeier geleitet. $^{46}$

42 Karl Bosl: Geschichte Bayerns. Band I: Vorzeit und Mittelalter. München 1952, S. 51.

43 Ebd., S. 65.

44 Goez (wie Anm. 17), S. 150 ff.

45 Bader (wie Anm. 31), S. 65.

46 Schmid (wie Anm. 11), S. 65. 
Die Krypten von Bleurville, Roßtal, Regenbach und anderswo waren „als Unterkirche für die Verehrung von Reliquien gedacht ${ }^{\text {“ }}{ }^{47}$ Pilger konnten dort ungestört die Heiligen verehren, während im darüberliegenden Gotteshaus Messen gelesen und Gebetszeiten eingehalten wurden. Je mehr Reliquien vorhanden waren, desto größer war die Anziehungskraft einer Stätte, an der Heilige fühlbar, sichtbar anwesend waren.

Im Fundmaterial von Regenbach hat sich ein Inschriftenstein erhalten, dessen Inschrift ${ }^{48}$ von Fachleuten gedeutet werden konnte: PRO SALVTE ANIMAE ILLIVS PER QVEM PERVENERVNT CORPORA SANCTORVM BEATORVM (Zum Seelenheil jenes Mannes, durch den die Körper der Heiligen hierher kamen). Diese Widmung beziehe ich auf Erzbischof Poppo von Trier. Zwar haben die Schriftexperten die Epoche, in welcher der Inschriftenstein entstanden ist, auf die Phase „Zwischen 800 und 1000 n. Chr.“ eingegrenzt. ${ }^{49}$ Aber warum sollte diese Inschrift nicht auch ein wenig später - um 1025/30 - entstanden sein?

Von Erzbischof Poppo weiß man, dass er die Reliquien des Priesters Beatus 1018 von Trier auf den Beatusberg bei Koblenz überführt hat. ${ }^{50}$ In Begleitung seines Freundes Simeon reiste Poppo in der Zeit um 1029 ins Heilige Land. ${ }^{51}$ Jerusalem wurde besucht. Man zog bis Ägypten, wo im Kairoer Vorort Babylon einst die Heilige Familie auf ihrer Flucht nach Ägypten gerastet hatte. Von einer solchen Pilgerfahrt ins Heilige Land kam ein Erzbischof gewiss nicht mit leeren Händen zurück. Da mag manche Reliquie ihren Weg auch an die Jagst gefunden haben. Die wichtigste Reliquie für Regenbach musste freilich nicht so weit reisen. Ihr Weg von Lauffen am Neckar nach Regenbach an der Jagst war überschaubar. Auch war man sich nicht fremd, denn bei der Heiligen Reginswindis handelte es sich um ein Mitglied der Sippe der Ernestinger. ${ }^{52}$

Anlässlich eines Aufenthaltes in Augsburg im Jahr 832 soll Kaiser Ludwig der Fromme dem treuen Nordgau-Grafen Ernst das Königsgut Lauffen am Neckar anvertraut haben. Die Nähe zu den Karolingern wird später noch intensiver, als ein Enkel Ludwigs des Frommen, Karlmann, mit einer namentlich nicht bekannten Tochter des Grafen Ernst verheiratet wird. ${ }^{53}$ Von einer weiteren Tochter des Grafen Ernst, dem Stammvater der Ernestinger, erfahren wir aus einer Heiligen-Vita, die freilich erst Jahrhunderte später - vermutlich Anfang des 13. Jahrhunderts - verfasst wird. ${ }^{54}$ Sie erzählt, dass dem Ernst und seiner Ehefrau in Lauffen ein Mädchen geboren wird: Reginswindis. Ihre Erziehung über-

47 Hartmut Schäfer, Günter Stachel: Unterregenbach. Archäologische Forschungen 1966-1988 (Archäologische Forschungen aus Baden-Württemberg 9). Stuttgart 1989, S. 27.

48 Ebd., S. 85.

49 Landesdenkmalamt Baden-Württemberg: Das Rätsel von Regenbach (Ausstellungskatalog). Stuttgart 1980, S. 18.

50 Schmid (wie Anm. 11), S. 20 (Anm. 38).

51 Ebd., S. 26.

52 Schwarzmaier (wie Anm. 8), S. $163 \mathrm{ff}$.

53 Ebd., S. 171.

54 Ebd., S. 169. 
nimmt eine Amme, deren Bruder auf dem Gut Lauffen als Knecht arbeitet. Dieser Knecht wird eines Tages - so sieht es jedenfalls die Amme - vom Grafen Ernst ungerecht behandelt. Aus Rache erwürgt sie daher das ihr anvertraute siebenjährige Kind und wirft den Leichnam in den Neckar. Drei Tage später wird die tote Reginswindis im Rachen eines Fisches steckend aufgefunden, ihre Gesichtsfarbe noch ganz frisch, grad so, als sei sie noch am Leben. Vom Volk ob ihres Martyriums als Heilige erkannt, soll ihr eine Verehrungsstätte in Lauffen geweiht werden.

„Am 13. Juli eines [...] nicht feststehenden Jahres weiht Bischof Hunbert die Reginswindiskirche zu Lauffen unter Transferierung der Gebeine der Heiligen dorthin" ${ }^{55}$ Wahrscheinlich geschah dies 839, denn in diesem Jahr fiel der 13. Juli auf einen Sonntag. Als Gedenktag aber wurde der 15. Juli festgelegt. Zukünftig erfuhr Reginswindis eine regionale Verehrung als Diözesan-Heilige im Bistum Würzburg. Darüber hinaus fand ihr Heiligentag Eingang in die Liturgie des Klosters Reichenau, ${ }^{56}$ also dort, wo der Babenberger Luitpold und die Ernestingerin Richwara für sich und ihre Nachkommen ein immerwährendes Gebetsgedenken gesichert hatten.

Erst nach der Jahrtausendwende hören wir erneut von Lauffen und seiner Heiligen. ${ }^{57}$ König Heinrich II. schenkt dem Bischof Heinrich von Würzburg $(† 1018)$ das Königsgut Kirchheim am Neckar in der Absicht, in LOUFEN UBI STA REGINSUINDIS VIRGO CORPORE REQUIESCIT (also dort, wo der Körper der Heiligen Reginswindis ruht) ein Kloster zur dauerhaften Verehrung der Heiligen zu errichten. Freilich gibt es keinerlei Hinweis, dass diese Verehrungsstätte damals in Lauffen entstanden ist. Erst mehr als 200 Jahre später erfuhr die Verehrung der Heiligen in Lauffen einen erneuten Aufschwung.

Ich vermute Folgendes: Kurz nachdem König Heinrich II. mit dem Würzburger Bischof die Errichtung einer klösterlichen Verehrungsstätte für Reginswindis vereinbart hatte, kam es zu lang anhaltenden Verstimmungen zwischen beiden, da der Herrscher einen Teil der Würzburger Diözese abtrennte für die 1007 erfolgte Neugründung des Bistums Bamberg. Folge war, dass der verärgerte Bischof manchen Wunsch des Herrschers gerade nicht befolgte, so auch nicht die Pläne für Lauffen. Nun war aber Poppo von Babenberg Dompropst der neuen Stiftung Bamberg. Heinrich II. war so mit ihm vertraut, dass er ihm das Erzbistum Trier verschaffte und ihn 1016 persönlich zur Amtseinführung nach Trier begleitete. Als 1018 beim Tod Bischof Heinrichs der Plan zur Errichtung einer angemessenen Verehrungsstätte für die heilige Reginswindis in Lauffen noch immer nicht umgesetzt war, trat er damit an seinen Vertrauten Poppo von Trier heran. Dieser ließ sich das nicht zweimal sagen, zumal er gerade Pläne für Re-

55 Alfred Wendehorst: Das Bistum Würzburg. Die Bischofsreihe bis 1254 (Germania Sacra. Die Bistümer der Kirchenprovinz Mainz I). Berlin 1962, S. 42.

56 Schwarzmaier (wie Anm. 8), S. 178.

57 Ebd., S. 180. 
genbach entwickelte. Und das dürfte dann der Dreiklang gewesen sein, aus dem die große Basilika mit ihrer Krypta zu Regenbach an der Jagst ab etwa 1020 entstand:

1. Eine Memorialkirche für die so tragisch ums Leben gekommenen Familienmitglieder Markgraf Luitpold († 994) und Herzog Ernst I. († 1015).

2. Eine repräsentative Grablege für die fränkischen Babenberger, vor allem, nachdem das Herzogsamt für Schwaben problemlos von Ernst I. auf Ernst II. übergegangen war, sich also eine dauerhafte Amtsnachfolge abzeichnete.

3. Eine Verehrungsstätte für die Heilige Reginswindis, die Familienheilige der Ernestinger, nun zwar nicht in Lauffen am Neckar, aber doch auch nicht allzu weit von der Stätte ihres Martyriums entfernt.

Das ist Spekulation, gewiss. Aber die weiteren Ereignisse sprechen dafür, dass es sich so oder so ähnlich abgespielt hat.

Ein Zeitsprung von 200 Jahren bringt uns in das beginnende 13. Jahrhundert. Der junge Staufer Friedrich II. aus Sizilien hat sich als Herrscher des Heiligen Römischen Reiches durchgesetzt. In Regenbach bröselte die große Basilika. Der Verfall war so weit vorangeschritten, dass man den westlichen Teil um 1200 ganz aufgab, das Kirchenschiff erheblich verkürzte und eine neue, bloß provisorisch errichtete Westfassade einzog. ${ }^{58}$ Man achtete jedoch darauf, dass die $\mathrm{Zu}$ gänge zur Krypta weiterhin geschützt waren. Auch wenn der Kirchenraum für Gottesdienste nicht mehr taugte, die Verehrung der Reliquien in der Krypta sollte weiterhin gesichert sein. Um die Jahrhundertmitte aber wurde auch der verbliebene Teil der Basilika abgebrochen. Nun war der einst so stattliche Kirchenbau nach 200 Jahren aus dem Ortsbild von Regenbach verschwunden.

In Lauffen am Neckar ist um 1212/19 das Geschlecht der Grafen von Lauffen im Mannesstamm ausgestorben. König Friedrich II. zog die Reichslehen ein und verpfändete dem Markgrafen Hermann V. von Baden die Städte Eppingen, Sinsheim und Lauffen. Und plötzlich erfuhr die Reginswindis-Verehrung am Neckar eine neue Blüte. ${ }^{59}$ Eine Inschrift auf einer altertümlichen Tumba verkündet sogar präzise: ANNO DOMINI MILLESIMO DUCENTESIMO VICESIMO SEPTIMO FUIT CANONISATA ET TRANSLATA VIRGO ET MARTIR SANCTA REGISWINDIS ET FUNDATA ECCLESIA. Im Jahre 1227 also soll die heilige Reginswindis ihre neue Verehrungsstätte bezogen haben.

Die damaligen Markgrafen von Baden waren Nachkommen der Richwara, Tochter von Herzog Hermann IV. von Schwaben $(\dagger 1038)$, haben also auch Wurzeln bei den Ernestingern und haben in der Zeit, als sie Lauffen in Besitz hatten, die Reliquien ihrer Familien-Heiligen offenbar wieder von der Jagst an den Neckar zurückgeholt.

58 Schäfer/Stachel (wie Anm. 47), S. 35.

59 Schwarzmaier (wie Anm. 8), S. 191/192. 
Im Zusammenhang damit dürfte auch die ursprüngliche Fassung der Vita von Reginswindis entstanden sein. Auffällig ist das Wunder, das der Heiligen dort zugeschrieben ist: Ihre Eltern seien nach ihrem Tod wieder in die alte bairische Heimat zurückgekehrt, hätten auch versucht, den Leichnam ihrer Tochter mitzunehmen - ,zu stehlen“ -, doch sei dieser Frevel gescheitert, da die Heilige am Ort ihres Martyriums ruhen wollte. Das mag man interpretieren als Antwort darauf, dass die Reliquien eben um 1020 für Regenbach ,gestohlen“ worden waren, nun aber ein für allemal in Lauffen zu verbleiben hätten. Bei diesem Reliquienschatz dürften jedoch in der Zwischenzeit Verluste aufgetreten sein. Denn die Verehrung der heiligen Reginswindis war nun auch auf der nahegelegenen Komburg zu bemerken.

Dort entstand 1220 die sogenannte Sechseck-Kapelle, die Reliquienkapelle des Klosters Komburg. In ihr hat Eduard Krüger 1940 eine romanische Wandmalerei freigelegt, ${ }^{60}$ die den Gekreuzigten zeigt, zu seinen Füßen ein Stifterpaar, die Frau auf der heraldisch rechten Seite, ein Ritter auf der heraldisch linken. Meines Erachtens handelt es sich bei der Stifterin um Adelheid von Gundelfingen, ${ }^{61}$ die zunächst mit Heinrich von Hohenlohe $(† 1215)$ verheiratet war, danach mit Konrad von Lobenhausen-Werdeck. Letzterer ist im Frühjahr 1221 unter den Rittern gewesen, die von Tarent/Italien auf einen Kreuzzug ins Heilige Land aufgebrochen sind. Die Sechseckkapelle der Komburg wurde vielleicht von Adelheid gestiftet, um eine gesunde Rückkehr ihres zweiten Ehemannes zu erflehen. Natürlich ist sie als Reliquienkapelle auch so konzipiert, dass sie nach der Rückkehr des Kreuzritters mitgebrachte Reliquien aus dem Heiligen Land hätte aufnehmen können. Ob Konrad gesund zurückgekehrt ist, lässt sich nicht mit Bestimmtheit sagen. Aber unter den späteren Reliquien der Sechseckkapelle dürften mit ziemlicher Sicherheit welche aus Regenbach gewesen sein. Ein Sohn Adelheids aus ihrer ersten Ehe war Gottfried von Hohenlohe ( $† 1254)$. Durch seine Heirat mit Richza von Krautheim dürften die Ansprüche entstanden sein, die ihm spätestens 1232 die Herrschaft über Langenburg und Regenbach gesichert haben. Etwa zu dieser Zeit könnten Reliquien der Heiligen Reginswindis nicht nur nach Lauffen, sondern auch in die von seiner Mutter gestiftete Reliquienkapelle auf der Komburg abgegeben worden sein.

Vor dem Bau der Kapelle war auf der Komburg am 15. Juli der Tag der Teilung der Apostel gefeiert worden, ${ }^{62}$ also die Aussendung der Apostel nach Pfingsten zur Missionierung der Völker. In späteren Jahrhunderten feierte man auf der Komburg jedoch neben Allerheiligen, Nikolaus (Kirchenpatron), Kirchweih (Thomas-Tag, 21. Dezember) und dem Frauentag zwischen den Schnitten (Ma-

60 Eduard Krüger: Schwäbisch Hall. Ein Gang durch Geschichte und Kunst. Neubearbeitung Schwäbisch Hall 1990, S. 144.

61 Heinz Bühler: Die Edelherren von Gundelfingen-Hellenstein. In: Jahrbuch des Historischen Vereins Dillingen 73 (1971), S. 13-40, hier S. 20.

62 Wolfgang Irtenkauf: Vom Kalender der Komburg im 12. Jahrhundert. In: Haalquell. Blätter für Heimatkunde des Haller Landes 13 (1961), Nr. 6. 
riä Himmelfahrt, 15. August) als eines von fünf Hochfesten ${ }^{63}$ im Jahreszyklus den 15. Juli als den Sankt Rensis-Tag oder Reynsen-Tag, hinter dessen Bezeichnung der Name der Heiligen Reginswindis nur noch mühsam zu erkennen ist. Der Verehrung von Heiligen in düsteren Krypten setzte die Schaulust der beginnenden Gotik nun Heiltumsweisungen im hellen Tageslicht entgegen. Die Sechseck-Kapelle der Komburg ist eines der wenigen baulichen Zeugnisse, die noch heute aufzeigen, wie diese Heiligenverehrung nun vollzogen wurde. Unter der Kapelle hindurch, in der Reliquien der Heiligen in kostbaren Schaugefäßen ruhten, führen Stufen hinauf zur Kirche. Der gläubige Pilger konnte sicher sein, dass der Segen, den er sich von den Heiltümern erhoffte, auf ihn ausstrahlte, sobald er unter der Reliquienkammer die Stufen hinaufschritt. Die Kapelle selbst ist von einer Galerie umgeben. Wenn an Hochfesten die Mönche des Klosters Komburg die Reliquien aus der Kapelle herausholten, schritten sie in immer neuen Prozessionen auf dieser Galerie rundum, während ein „Heiltumsplärrer“ die gerade gezeigte Reliquie den in Verzückung zur Galerie hochblickenden Gläubigen beschrieb.

Solch glorreichem Schauspiel hatte eine düstere Krypta von Regenbach nichts entgegenzusetzen. „Der Abbruch (der großen Basilika) dürfte bis zur Mitte des 13. Jahrhunderts erfolgt sein“ ${ }^{64}$ Die Steine könnten für den Ausbau der Burg Langenburg verwendet worden sein. Die Krypta verlor ihre Funktion. Als Kellerraum für ein darüber errichtetes Gebäude hat sie die Jahrhunderte überstanden.

Die österreichischen Babenberger starben 1246 im Mannesstamm aus. Der letzte männliche Vertreter der fränkischen Babenberger könnte Abt Hermann († 1065) vom Kloster Einsiedeln gewesen sein. Die Töchter dieser Linie hatten ihre Nachkommen in den Zähringern, den Markgrafen von Baden, den Markgrafen von Vohburg, den Grafen von Andechs-Dießen. Über Eilika, die Schwester des in der Urkunde für Regenbach zeugenden Otto von Schweinfurt, ging Erbe nach Sachsen, von dort bis in den Hennegau. In diesen Sippen wird das Wissen um die frühe Geschichte ihrer Vorfahren mit der Zeit verblasst sein. Einer freilich ist dauerhaft in die Sagenwelt der deutschen Überlieferungen eingegangen: der stürmische Herzog Ernst II., der so aufmüpfig und voll jugendlicher Rauflust um das familiäre Erbe gekämpft hatte. Der Adelsgesellschaft des Mittelalters imponierte das, später auch den bürgerlichen Romantikern.

Am 25. September 1819 tauschten im Schloss zu Ludwigsburg Ständeversammlung und König ihre Exemplare der Verfassung, nach der das Leben im Königreich Württemberg zukünftig organisiert sein sollte. Für den 6. Dezember war die Eröffnung des Landtags vorgesehen. Dazwischen aber, am 29. Oktober, wollten die Bürger, die um diese neue Verfassung gestritten hatten, im Hof- und

63 Friedrich Pietsch (Bearbeiter): Die Urkunden des Archivs der Reichsstadt Schwäbisch Hall. Bd. 1 (1156-1399). (Veröffentlichungen der Staatlichen Archivverwaltung Baden-Württemberg 21). Stuttgart 1967, S. 240, U 786.

64 Schäfer, Stachel (wie Anm. 47), S. 37. 
Nationaltheater zu Stuttgart feiern. Gegeben wurde „Ernst Herzog von Schwaben“. Ludwig Uhland schrieb im Vorfeld noch eigens einen Prolog. ${ }^{65}$

„Ein ernstes Spiel wird euch vorübergehn, der Vorhang hebt sich über einer Welt, die längst hinab ist in der Zeiten Strom...“

Jahrhunderte nach seinem Tod war Herzog Ernst II. mal wieder in aller Munde. Doch niemand sprach von Regenbach.

\section{Anlage: Die fränkischen Babenberger. Versuch einer Genealogie}

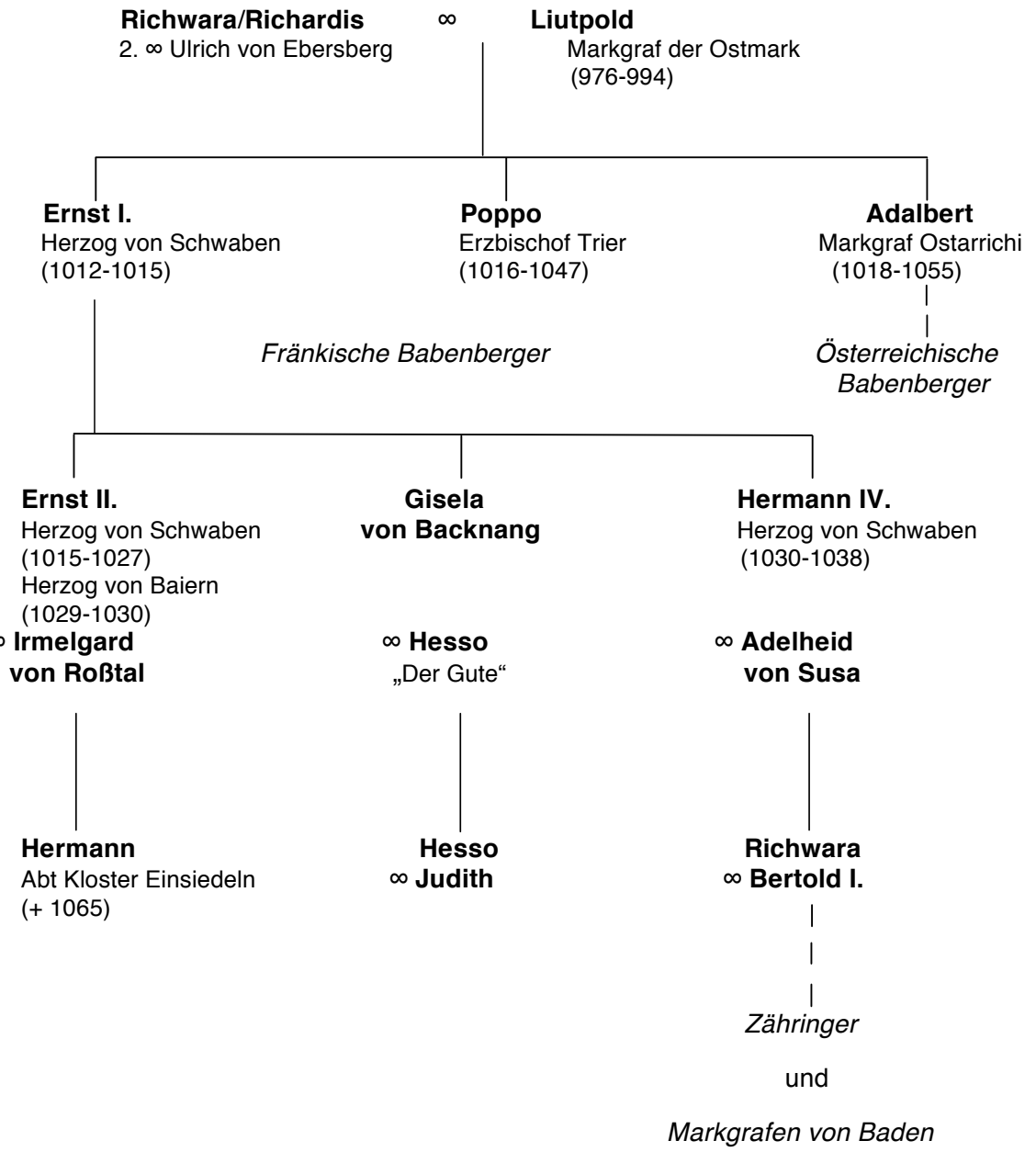

65 Hartmut Fröschle/Walter Scheffler (Hg.): Ludwig Uhland Werke. Bd. I: Sämtliche Gedichte. S. 76 . 